\title{
High co-expression of TNF- $a$ and CARDS toxin is a good predictor for refractory Mycoplasma pneumoniae pneumonia
}

Gang Li ${ }^{\dagger}$, Liping Fan ${ }^{\dagger}$, Yuqing Wang ${ }^{\dagger},{ }_{*}$ Li Huang, Meijuan Wang, Canhong Zhu, Chuangli Hao, Wei Ji, Hansi Liang, Yongdong Yan $^{*}$ and Zhengrong Chen ${ }^{*}$

\begin{abstract}
Background: Early distinction between refractory M. pneumoniae pneumonia (RMPP) and non-RMPP (NRMPP) is still difficult. The community-acquired respiratory distress syndrome (CARDS) toxin can induce inflammatory and histopathological phenotypes associated with M. pneumoniae infection. This study aimed to investigate the clinical significance of CARDS toxin and pro-inflammatory cytokines in children with RMPP and to explore whether CARDS toxin can induce TNF-a expression.

Methods: Levels of CARDS toxin and cytokines in BALF from control and children with MPP were determined by real-time PCR and ELISA, respectively. A receiver-operating characteristic (ROC) analysis was performed to assess the diagnostic values of CARDS toxin, TNF-a, and IL-6 in RMPP. The recombinant CARDS toxin was constructed and prepared at different concentrations for stimulation of RAW264.7 cells. After co-culture with CARDS toxin, cytokines were detected by ELISA and the mRNA levels were measured by real-time PCR. Effects of CARDS toxin and TNF-a on inflammatory cell infiltration and mucus secretion in mouse lungs were also evaluated.

Results: Levels of CARDS toxin, TNF- $a$ and IL-6 in bronchoalveolar lavage fluid (BALF) were significantly higher in RMPP cases compared with NRMPP cases. Furthermore, TNF-a had better diagnostic ability for differentiation of RMPP with AUC of 0.824 and Youden index of 0.692 compared with CARDS toxin and IL-6. Moreover, CARDS toxin was positively correlated with TNF-a level in MPP cases. In vitro assay revealed that CARDS toxin induced RAW264.7 macrophages to secrete TNF-a. Further in vivo assay showed that TNF-a deletion partially abrogated the CARDS toxin-mediated induction of inflammatory cell infiltration and mucus secretion in mouse lungs.
\end{abstract}

Conclusions: The high co-expression of TNF-a and CARDS toxin in BALF is a good diagnostic biomarker for differentiating children with RMPP and NRMPP.

Keywords: TNF-a, CARDS toxin, M. pneumoniae pneumonia, RMPP

\section{Background}

Mycoplasma pneumoniae pneumonia (MPP) caused by Mycoplasma pneumoniae (M. pneumoniae) infection is one of the most common forms of community-acquired pneumonia (CAP) in children (Wang et al., 2005; Gao et al., 2018). Although M. pneumoniae infection is usually considered as a self-limited process, sometimes it may cause various severe complications such as arthritis and

\footnotetext{
*Correspondence: Yan_yongdong@126.com; chen_zheng_rong@163.com ${ }^{\dagger}$ Gang Li, Liping Fan and Yuqing Wang contributed equally to this work. Department of Respiratory medicine, Children's Hospital of Soochow University, Jingde Road NO.303, Suzhou 215003, Jiangsu Province, China
}

necrotizing pneumonia (Zhang et al., 2016; Barreira et al., 2009; Azumagawa et al., 2008). Macrolides are the firstchoice antibiotics for M. pneumoniae infections for children (Zhang et al., 2016). However, there are still some cases showing clinical and radiological deterioration in spite of macrolide antibiotic therapy for 7 days or longer, which was defined as refractory M. pneumoniae pneumonia (RMPP) (Zhang et al., 2016). The causes of RMPP are multiple, which include macrolide resistance, infections with other pathogen and immune disorder (Zhang et al., 2016; Barreira et al., 2009; Azumagawa et al., 2008; Ding et al., 2018). RMPP cases present with more frequent

(C) The Author(s). 2019 Open Access This article is distributed under the terms of the Creative Commons Attribution 4.0 International License (http://creativecommons.org/licenses/by/4.0/), which permits unrestricted use, distribution, and 
occurrences of cough, fever, and abnormal lung signs compared with non-RMPP (NRMPP) cases, and can develop into a severe life-threatening pneumonia (Ding et al., 2018). Therefore, it is important for pediatricians to recognize RMPP earlier and treat it promptly and prevent the progress of the disease.

M. pneumoniae, a leading pathogen causing CAP in children, shows cytotoxicity through expressing some pathogenic factors including community-acquired respiratory distress syndrome (CARDS) toxin (MPN372) (Becker et al., 2015). CARDS toxin is a 591-amino-acid ADP-ribosylating and vacuolating protein (Shimizu, 2016). CARDS toxin can induce and mimic major inflammatory and histopathological phenotypes associated with M. pneumoniae infection in rodents and primates (Ramasamy et al., 2018). However, CARDS toxin has currently only been studied in animal experiments, and there are few clinical studies concerning the correlation between CARDS toxin and children with MPP, especially RMPP.

A previous study has demonstrated that both mice and baboon responded to exposure to recombinant CARDS toxin by increasing the expression of the pro-inflammatory cytokines such as Interleukin (IL)- 6 and tumor necrosis factor (TNF)- $\alpha$ (Hardy et al., 2009). TNF- $\alpha$ is mainly produced by monocytes and macrophages, and participates in the lung injury associated with severe pneumonia (Ding et al., 2018). Recent studies have shown several new biomarkers used for etiologic diagnosis in children with CAP (Principi \& Esposito, 2017; Sungurlu \& Balk, 2018; Liu et al., 2018). Some of these biomarkers have also been used for evaluating the M. pneumoniae infection severity (Chkhaidze \& Kapanadze, 2017). Serum level of TNF- $\alpha$ has shown to be significantly higher in children with MPP compared with healthy children (Wang et al., 2005). However, whether TNF- $\alpha$ can be used as a biomarker for RMPP has not been defined.

Therefore, this study aimed to investigate the clinical significance of CARDS toxin and pro-inflammatory cytokines including TNF- $\alpha$ in bronchoalveolar lavage fluid (BALF) and explored predicting factors of RMPP in children. In addition, we explored whether the potential mechanism of TNF- $\alpha$ upregulation was associated with CARDS toxin.

\section{Methods}

\section{Patients and study design}

From January 2015 to June 2016, cases with MPP confirmed byboth positive enzyme-linked immunosorbent assay (ELISA) and polymerase chain reaction (PCR) were enrolled $(N=71)$. All cases were from 1 month to 14 years old and had fever, cough, tachypnea, chest retractions, abnormal auscultatory findings and radiologic evidence of CAP. Cases were not included if they had chronic lung disease, immunodeficiency, bronchopulmonary malformation, or co-infection.

Among these 71 children with MPP, 21 were diagnosed with RMPP and 50 with NRMPP. The RMPP was defined as showing a prolonged high degree fever $\left(>38.5^{\circ} \mathrm{C}\right)$, radiological deterioration (increased infiltration area compared to first radiological finding) after macrolide combined oral prednisolone therapy (Azithromycin, $10 \mathrm{mg} / \mathrm{d}$ and Prednisolone, $1-2 \mathrm{mg} / \mathrm{d}$ ) for 7 days or more (Tamura et al., 2008). Other children were defined as NRMPP.

A total of 22 children with bronchial foreign bodies who underwent foreign body removal in our hospital at the same time were selected as the control group. The inclusion criteria of the control group were: They had (1) a clear history of foreign body inhalation and a history of an irritating cough; (2) no inflammatory changes suggested by chest radiographs; (3) no respiratory tract infection within 32 months; (4) no history of application of hormones and immunosuppressive agents.

The study was approved by the Ethics Committee of the Children's Hospital of Soochow University. Informed consent was obtained from parents or guardians of the children.

\section{Serology of M. pneumoniae}

The specific antibodies against $M$. pneumoniae (IgM and IgG) were detected in serum samples $(2 \mathrm{~mL})$ of children during the acute phase (upon admission) and convalescent phase (upon discharge) respectively using a commercial ELISA kit (Serion ELISA classic M. pneumoniae IgG/IgM, Institute Virion/Serion, Würzburg, Germany), according to the manufacturer's instructions. Acute $M$. pneumoniae infection was defined with either a single positive serum IgM titer or a 4-fold increase in IgG titer in the convalescent serum sample (Ding et al., 2018).

\section{Real-time fluorescent quantitation PCR for M. pneumoniae gene detection}

The procedure of PCR for M. pneumoniae 16S rRNA detection was performed as described previously (Wang et al., 2014). Briefly, after being shaken for $30 \mathrm{~s}$, one of the equally divided samples of BALF was centrifuged at 15 , $000 \times g$ for $5 \mathrm{~min}$. After that, the sediment was collected and extraction of DNA from a $400-\mu \mathrm{L}$ sample was performed. The DNA was then amplified using specific primers and probe. The sequences of the primers and probe were as follows: M. pneumoniae, forward: $5^{\prime}$ GCAAGGGTTCGTTATTTG-3'; reverse: 5'-CGCCTG CGCTTGCTTTAC-3' (amplicon size: 380 bp); M. pneumoniae-probe: 5'-AGGTAATGGCTAGAGTTTGACTG3'. The real-time PCR was performed using the iQ5TM BIO-icycler (Bio-Rad, Hercules, CA, USA). The PCR conditions were as follows: $37^{\circ} \mathrm{C}$ for $2 \mathrm{~min}$; initial denaturation at $94{ }^{\circ} \mathrm{C}$ for $10 \mathrm{~min}$, followed by 40 cycles of 
denaturation at $94{ }^{\circ} \mathrm{C}$ for $10 \mathrm{~s}$, annealing at $55^{\circ} \mathrm{C}$ for $30 \mathrm{~s}$, and extension at $72^{\circ} \mathrm{C}$ for $40 \mathrm{~s}$. Quantification curves were plotted using standard control samples at several concentrations (Daan gene Co. Ltd., Guangzhou, China). For each assay, a negative quality control, a critical quality control, a positive quality control, and four positive quantity controls $\left(10^{5}\right.$ copies $/ \mathrm{mL}, 10^{6}$ copies $/ \mathrm{mL}, 10^{7}$ copies/ $\mathrm{mL}$, and $10^{8}$ copies $/ \mathrm{mL}$ ) were used.

\section{Collection of clinical data}

The age, sex, the duration of hospital stay and fever, the occurrence of pleural effusion, mucus plug and complications, the laboratory test data, and the effectiveness of treatment with macrolide were collected upon hospital admission and at patient discharge (Tables 1 and 2). All MPP children enrolled presented with or without mucus plug and pleural effusion on chest radiography.

Peripheral venous blood $(2-3 \mathrm{~mL})$ was collected within $24 \mathrm{~h}$ from children in each group. The blood sample was immediately sent for the following laboratory examination: the complete blood count, C-reactive protein (CRP), lactate dehydrogenase (LDH), subpopulations of T lymphocytes, andspecific antibody to M. pneumoniae and other tests (Table 1).

Flexible fiber optic bronchoscopy and bronchoalveolar lavage were performed following the guidelines described previously (Wang et al., 2017a). The BALF was gently aspirated, collected and prepared for detection of $M$. pneumoniae DNA, CARDS toxin, and cytokines. The cytokines including TNF- $\alpha$, interferon- $\gamma$ (IFN- $\gamma$ ), IL-36, IL-17, IL-8, IL-6, and IL-4 in the supernatants of BALF were determined using their commercial ELISA kits (R\&D Systems, Minneapolis, MN, USA) according to the manufacturer's instructions (Table 2). All children were treated with alveolar irrigation and drainage using a fiberoptic bronchoscope as previously described (Xu et al., 2011).

\section{Real-time PCR}

The relative expression of CARDS toxin in BALF from children was determined by real-time PCR. Briefly, total RNA was extracted from BALF using TRIzol reagent (Invitrogen, Waltham, MA, USA) according to the manufacturer's protocol. RNA was then reverse transcribed into cDNAs using RNA Transcription Kit (Promega, USA). The cDNA templates were amplified by qRT-PCR using SYBR Green PCR Mix (TaKaRa, Japan). The primer sequences were as follows: CARDS toxin: forward, $5^{\prime}$-TTCC ACTTCAGAAACACCCACAGC-3', reverse, 5' - TCAA TCAGGGCACGCAAACG-3'; pdhA (internal control for CARDS toxin): forward, 5 -ACTGGTTCTGCCCTACCT TCCGTTCC-3', reverse, 5'-CTTCGTGCATTGCTTC GTAACTCGC-3'. The relative expression of CARDS toxin was calculated by the $2^{-\Delta \mathrm{Ct}}$ method and normalized to the internal control pdhA.

The mRNA level of TNF- $\alpha$ in RAW264.7 cells was determined by real-time PCR as previously described (Ma et al., 2016). The primer sequences were as follows: TNF- $\alpha$ : forward, 5' - AGCCGATGGGTTGTACCT-3',

Table 1 Demographic data and clinical characteristics in children with RMPP and NRMPP

\begin{tabular}{|c|c|c|c|c|c|c|}
\hline Clinical parameters & $\begin{array}{l}\text { MPP cases } \\
(n=71)\end{array}$ & $\begin{array}{l}\text { Controls } \\
(n=22)\end{array}$ & $P$ value & $\begin{array}{l}\text { RMPP } \\
(n=21)\end{array}$ & $\begin{array}{l}\text { NRMPP } \\
(n=50)\end{array}$ & $P$ value \\
\hline Age,months & $64.6 \pm 32.0$ & $62.0 \pm 29.2$ & 0.733 & $64.5 \pm 27.6$ & $64.6 \pm 34.0$ & 0.986 \\
\hline Male, n(\%) & $29(40.8)$ & $11(50)$ & 0.449 & $11(52.4)$ & $18(36)$ & 0.2 \\
\hline Hospital stay, days & $10.1 \pm 4.1$ & N/A & & $12.6 \pm 5.5$ & $9.0 \pm 2.8$ & $<0.001$ \\
\hline Duration of fever, days & $8.2 \pm 4.4$ & N/A & & $12.5 \pm 3.1$ & $6.4 \pm 3.4$ & $<0.001$ \\
\hline pleural effusion, n(\%) & $29(40.8)$ & N/A & & $16(76.2)$ & $13(26)$ & $<0.001$ \\
\hline mucus plug, n(\%) & $21(29.6)$ & N/A & & $15(71.4)$ & $6(12)$ & $<0.001$ \\
\hline expulmonary complications, n(\%) & $13(18.3)$ & N/A & & $7(33.3)$ & $6(12)$ & 0.047 \\
\hline $\mathrm{WBC}, 10^{6} / \mathrm{L}$ & $9.2 \pm 3.7$ & N/A & & $9.0 \pm 1.7$ & $9.2 \pm 4.2$ & 0.766 \\
\hline$C R P, m g / L$ & $14.9(4.7-34.9)$ & N/A & & $23.3(10.3-44.6)$ & $12.8(4.4-31.3)$ & 0.03 \\
\hline $\mathrm{LDH}, \mathrm{IU} / \mathrm{L}$ & $480.9 \pm 162.5$ & N/A & & $581.5 \pm 179.7$ & $437.2 \pm 134.4$ & 0.001 \\
\hline Lymphocytes in blood & & N/A & & & & \\
\hline $\mathrm{CD} 3+, \%$ & $67.0 \pm 9.4$ & N/A & & $68 \pm 8.2$ & $66.6 \pm 10.0$ & 0.595 \\
\hline $\mathrm{CD} 3+\mathrm{CD} 4+, \%$ & $36.1 \pm 9.3$ & N/A & & $35.3 \pm 7.6$ & $36.5 \pm 10.0$ & 0.654 \\
\hline $\mathrm{CD} 3+\mathrm{CD} 8+, \%$ & $26.4 \pm 7.2$ & N/A & & $27.3 \pm 6.0$ & $26.0 \pm 7.6$ & 0.494 \\
\hline CD3-CD $(16+56+), \%$ & $10.9 \pm 7.8$ & N/A & & $9.7 \pm 7.7$ & $11.4 \pm 7.8$ & 0.481 \\
\hline CD3-CD19+, \% & $19.9 \pm 8.5$ & N/A & & $20.1 \pm 7.2$ & $19.9 \pm 9.1$ & 0.904 \\
\hline
\end{tabular}


Table 2 Comparison of CARDS toxin and cytokines in BALF from control and MPP (including RMPP and NRMPP) children

\begin{tabular}{|c|c|c|c|c|c|c|}
\hline CARDS toxin and cytokines in BALF & $\begin{array}{l}\text { MPP cases } \\
(n=71)\end{array}$ & $\begin{array}{l}\text { Controls } \\
(n=22)\end{array}$ & $P$ value & $\begin{array}{l}\text { RMPP } \\
(n=21)\end{array}$ & $\begin{array}{l}\text { NRMPP } \\
(n=50)\end{array}$ & $P$ value \\
\hline CARDS toxin, relative expression & $12.5(4.9-31.1)$ & $0.5(0.2-1.4)$ & $<0.001$ & $19.6(9.0-63.0)$ & $10.0(3.1-25.5)$ & 0.025 \\
\hline TNF-a, pg/ml & $68 \pm 12.7$ & $40.4 \pm 6.2$ & $<0.001$ & $76.9 \pm 11.2$ & $64.2 \pm 11.4$ & $<0.001$ \\
\hline IFN- $\gamma, \mathrm{pg} / \mathrm{ml}$ & $541.2 \pm 104.1$ & $357.3 \pm 83.3$ & $<0.001$ & $535.6 \pm 96.9$ & $543.5 \pm 104.8$ & 0.772 \\
\hline IL-36, pg/ml & $429.9 \pm 60.6$ & $360.5 \pm 61.2$ & $<0.001$ & $438.8 \pm 61.6$ & $426.5 \pm 60.5$ & 0.457 \\
\hline IL-17, pg/ml & $23.6 \pm 5.6$ & $24.7 \pm 5.4$ & 0.428 & $23.6 \pm 5.4$ & $23.6 \pm 5.8$ & 0.986 \\
\hline IL-8, pg/ml & $67 \pm 15.0$ & $52.2 \pm 14.7$ & $<0.001$ & $65.4 \pm 14.7$ & $67.7 \pm 15.2$ & 0.584 \\
\hline IL-6, pg/ml & $24.7 \pm 5.7$ & $16.1 \pm 4.0$ & $<0.001$ & $28.1 \pm 3.8$ & $23.2 \pm 5.8$ & $<0.001$ \\
\hline $\mid \mathrm{L}-4, \mathrm{pg} / \mathrm{ml}$ & $66.8 \pm 14.6$ & $36.4 \pm 6.5$ & $<0.001$ & $68.7 \pm 12.5$ & $66.0 \pm 15.4$ & 0.477 \\
\hline
\end{tabular}

RMP refractory Mycoplasma pneumoniae pneumonia, NRMPP non-refractory Mycoplasma pneumoniae pneumonia, CARDS toxin community-acquired respiratory distress syndrometoxin, BALF bronchoalveolar lavage fluid, TNF- $a$ tumor necrosis factor- $a, I F N-\gamma$ interferon- $\gamma, I L-36$ interleukin-36, $L L-17$ interleukin-17, IL-8 interleukin-8, IL-6 interleukin-6, IL-4 interleukin-4

reverse, 5' - TGAGTTGGTCCCCCTTCT-3'; 18S: forward, 5' - GCCCGAGCCGCCTGGATAC-3', reverse, $5^{\prime}$ - CCGGCGGGTCATGGGAATAAC-3'. TNF- $\alpha$ mRNA level was calculated by the $2^{-\Delta \Delta C t}$ method and normalized to the internal control $18 \mathrm{~S}$.

\section{Construction of recombinant CARDS toxin}

The full-length gene sequence and protein sequence of MPN372 were obtained from the NCBI database. $M$. pneumoniae uses TGA to encode tryptophan and TGA is a termination codon in most other species. Therefore, we mutated eight codon TGAs encoding tryptophan into TGG. The optimized MPN372 gene sequence was cloned into the pFastBac donor plasmid vector for virus packaging. High-Five cells in logarithmic growth phase were infected with high titer recombinant virus to express the target protein, which was further purified by nickel column.

\section{Vacuolization induction by CARDS toxin}

HeLa cells were purchased from ATCC (Manassas, VA, USA) and cultured in RPMI-1640 medium supplemented with $10 \%$ fetal calf serum (FCS) in a $37{ }^{\circ} \mathrm{C}$ humidified incubator with air and $5 \% \mathrm{CO}_{2}$. When the cell confluence reached $60-70 \%$, the culture medium was replaced with fresh RPMI-1640 medium containing $50 \mu \mathrm{g} /$ $\mathrm{mL}$ CARDS toxin. The medium without CARDS toxin served as the negative control. After $24 \mathrm{~h}$ and $48 \mathrm{~h}$ of incubation, the ability of CARDS toxin to induce vacuolization in HeLa cells was evaluated under a microscope (Olympus BH-2; Olympus Corporation, Tokyo, Japan).

\section{RAW264.7 cell stimulation by CARDS toxin in vitro}

The murine macrophage cell line RAW264.7 was purchased from ATCC. RAW264.7 cells were cultured in RPMI-1640 medium, supplemented with $10 \%$ heat-inactivated FCS, $2 \mathrm{mML}$-glutamine, $100 \mathrm{U} / \mathrm{mL}$ penicillin, and $100 \mu \mathrm{g} / \mathrm{mL}$ streptomycin, at $37^{\circ} \mathrm{C}$ under $5 \% \mathrm{CO}_{2}$. The cell density was adjusted to $2 \times 10^{5}$ cells $/ \mathrm{ml}$ in 12 -well plates. When the cell confluence reached about $50 \%$, the culture medium was replaced with fresh RPMI-1640 medium containing different concentrations of CARDS toxin $(0,5$, and $50 \mu \mathrm{g} / \mathrm{mL}$ ). After $48 \mathrm{~h}$ of incubation, the cells were harvested and the levels of 13 cytokines including TNF- $\alpha$, IFN- $\gamma$, IL-2, IL-4, IL-5, IL-6, IL-9, IL-10, IL-13, IL-17A, IL-17F, IL-21, and IL-22 in the supernatant were measured using LEGENDplex ${ }^{\mathrm{Tm}}$ Mouse Th Cytokine Panel (13plex) (BioLegend, San Diego, CA, USA) according to the manufacturer's instructions.

\section{Animals and in vivo experiments}

The animal used in this experiment were approved by the Ethics Committee of Children's Hospital of Soochow University. BALB/c mice (age of 6-7 weeks, weighing $20 \pm 2 \mathrm{~g}$ ) were purchased from the Laboratory Animal Center of Soochow University, Suzhou, China. TNF- $\alpha^{-1-}$ mice (BALB/c background) were purchased from Jackson Laboratory (Bar Harbor, ME, USA). All animals were kept in separate cages and housed under constant temperature $\left(25 \pm 1{ }^{\circ} \mathrm{C}\right.$ ) and humidity (50\%) with $12 \mathrm{~h}$ light-dark cycles, and had free access to food and water.

CARDS toxin $(50 \mu \mathrm{g} / 0.5 \mathrm{~mL})$ or equivalent PBS was intratracheally instilled into wild-type $\mathrm{BALB} / \mathrm{c}$ mice and TNF- $\alpha^{-1-}$ mice ( $N=5 /$ group). On the 7 th day, the mice were sacrificed by cervical dislocation under anesthesia and lung tissues were harvested for histological examination.

\section{Histological examination}

Heamatoxylin and eosin (H\&E) staining was performed to observe the infiltration of inflammatory cells in the lungs. Periodic acid-Schiff (PAS) staining was performed to observe the secretion of bronchial mucus. Briefly, mouse lung tissues were fixed with $4 \%$ formaldehyde, embedded in paraffin and cut to obtain serial 4- $\mu \mathrm{m}$ thick sections. Subsequently, these sections were stained with H\&E (Yeasen, Shanghai, China) and PAS (Solarbio, 
Beijing, China) following routine staining procedures. The pathologic changes in these tissues were observed under a light microscope (Olympus BH-2; Olympus Corporation, Tokyo, Japan). H\&E average score and the percentage of PAS-positive $\left(\mathrm{PAS}^{+}\right)$epithelial cells were analyzed by a pathologist in a blinded fashion.

\section{Statistical analysis}

All analyses were performed using SPSS for Windows (version 21.0; SPSS Inc., Chicago, IL, USA). Categorical data were analyzed using the Chi-square test. Continuous variables were analyzed using Student'st-test or the MannWhitney U-test if the data had a non-normal distribution. For in vitro studies, statistical significance was determined using one-way ANOVA. Correlations between CARDS toxin and TNF- $\alpha$ or IL- 6 were evaluated by Spearman correlation test. Data were expressed as means \pm standard deviation (SD) if data had a normal distribution. $p<0.05$ was considered statistically significant.

\section{Results}

Demographic data and clinical characteristics in children with RMPP and NRMPP

As shown in Table 1, there was no significant difference in mean age and gender between control and MPP children. Of note, the RMPP cases had longer hospital stays and duration of fever than the NRMPP cases (all $p<0.05$ ). The occurrences of pleural effusion, mucus plug, and expulmonary complications were more frequent in RMPP cases when compared with NRMPP cases (all $p<0.05)$. Furthermore, levels of CRP and LDH in peripheral blood were significantly higher in RMPP cases compared with NRMPP cases $(p<0.05)$. However, there was no significant difference in WBC and lymphocytes distributions between RMPP and NRMPP cases.

\section{Comparison of CARDS toxin and cytokines in BALF from control and MPP children}

As shown in Table 2, the relative expression of CARDS toxin and levels of TNF- $\alpha$, IFN- $\gamma$, IL-36, IL-8, IL-6, and IL4 in BALF from MPP cases were significantly higher when compared with control cases (all $p<0.05$ ). However, there was no significant difference in IL-17 levels in BALF between the control and MPP cases. Furthermore, the relative expression of CARDS toxin and levels of TNF- $\alpha$ and IL-6 in BALF were significantly higher in RMPP cases compared with NRMPP cases (all $p<0.05$ ). However, there was no significant difference in levels of IFN- $\gamma$, IL-36, IL-8, IL-4, and IL-17 in BALF between RMPP and NRMPP cases.

\section{Comparison of CARDS toxin expression between MPP cases with/without plug and pleural effusion}

Data revealed that the expression of CARDS toxin was significantly higher in BALF from children with MPP with mucus plug than that in children with MPP without plug ( $p=0.006$; Fig. 1a). However, no significant difference in CARDS toxin was observed in BALF from children between MPP with pleural effusion and MPP without pleural effusion (Fig. 1b).

\section{CARDS toxin was positively correlated with TNF-a level in MPP cases}

We next analyzed the correlations between CARDS toxin and pro-inflammatory cytokines TNF- $\alpha$ and IL-6 in BALF from children with MPP. Data showed that TNF- $\alpha$ level was positively correlated with CARDS toxin

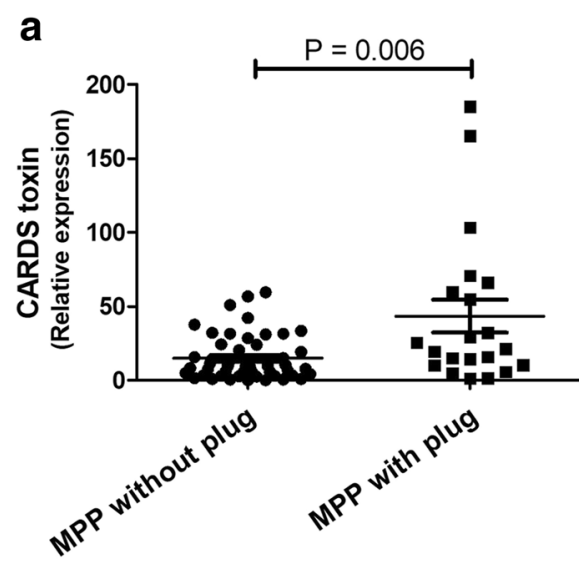

b
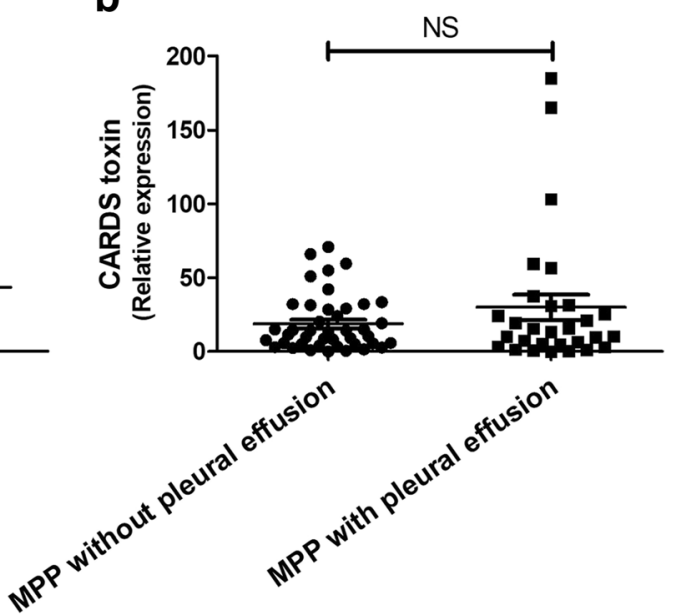

Fig. 1 Comparison of CARDS toxin expression between MPP cases with/without plug (a) and pleural effusion (b). Children with MPP were divided into MPP with plug group ( $N=50)$ and MPP without plug group $(N=21)$, according to the presence or absence of mucus plugs. They were also divided into MPP with pleural effusion group $(N=42)$ and MPP without pleural effusion $(N=29)$ group, according to results of imageological examination. The expression of CARDS toxin in bronchoalveolar lavage fluid (BALF) in each group was measured. NS, no significance 
level $(\mathrm{r}=0.418, p=0.0003$; Fig. 2a). In contrast, IL-6 level was negatively correlated with CARDS toxin level ( $\mathrm{r}=-0.2152, p=0.072$; Fig. 2b).

\section{Diagnostic values of CARDS toxin, TNF- $a$, and IL- 6 in children with RMPP}

To estimate the diagnostic abilities of CARDS toxin, TNF$\alpha$, and IL- 6 in children with RMPP, a receiver-operating characteristic (ROC) analysis was performed. As shown in Fig. 3, TNF- $\alpha$ had better diagnostic ability for differentiation of RMPP with the best cut-off of $68.25 \mathrm{pg} / \mathrm{ml}$, AUC of 0.824 and Youden index of 0.692 compared with CARDS toxin and IL-6.

\section{Plasmid construction and vacuolation activity verification of CARDS toxin}

The recombinant CARDS toxin was constructed (Fig. 4a) and the purity of CARDS toxin was checked by SDSPAGE (Fig. 4b). The bioactivity of CARDS toxin was determined by the toxin's ability to induce vacuolization in HeLa cells. Compared with the control group, CARDS toxin exhibited the ability to induce vacuolization in HeLa cells (Fig. 4c). These results confirmedthe bioactivity of CARDS toxin based on the ability to produce vacuoles in epithelial cells.

\section{CARDS toxin induced RAW264.7 macrophages to secrete TNF-a}

The levels of 13 cytokines including TNF- $\alpha$, IFN- $\gamma$, IL-2, IL-4, IL-5, IL-6, IL-9, IL-10, IL-13, IL-17A, IL-17F, IL21 , and IL-22 in the supernatant of CARDS toxin-stimulated RAW264.7 cells were measured using ELISA. Data revealed that CARDS toxin significantly increased TNF$\alpha$ level secreted from RAW264.7 macrophages in a dose-dependent manner $(p<0.05)$, whereas it had no significant effect on levels of the other cytokines (Fig. 5a). The data of real-time PCR further confirmed that TNF$\alpha$ mRNA level in RAW264.7 cells was notably upregulated by $50 \mu \mathrm{g} / \mathrm{mL}$ CARDS toxin ( $p<0.05$; Fig. $5 \mathrm{~b}$ ).
Effect of CARDS toxin on inflammatory cell infiltration and mucus secretion in mouse lungs

As shown in H\&E staining, there was no obvious inflammatory cell infiltration around the airway and vascular tissue (Fig. 6a). Compared with the PBS group, the wild type mice treated with CARDS toxin showed airway tube cavity stenosis and a large amount of inflammatory cell infiltration around the airway and vascular tissue (Fig. 6b). Compared with wild type mice, the TNF- $\alpha^{-1-}$ mice treated with CARDS toxin showed a notable decrease in inflammatory cell infiltration (Fig. 6c).

As indicated in PAS staining, there were few PAS-positive epithelial cells in the airway in the PBS group (Fig. 6d). Compared with the PBS group, the wild type mice treated with CARDS toxin showed a significant increase in PAS-positive epithelial cells (i.e. goblet cell hyperplasia) and hyperactive mucus secretion (Fig. 6e). Compared with wild type mice, the TNF- $\alpha^{-1-}$ mice treated with CARDS toxin showed a notable decrease in PAS-positive epithelial cells (Fig. 6f). Taken together, these data indicated that TNF- $\alpha$ is involved in the CARDS toxin-mediated induction of inflammatory cell infiltration and mucus secretion in mouse lungs.

\section{Discussion}

Although most of the children with $M$. pneumoniae infection could make remarkable recovery, the prevalence of RMPP has rapidly increased in recent years due to the abuse of macrolides and the emergence of antibioticresistant strains (Wang et al., 2017b). Thus, it is important for pediatricians to recognize RMPP earlier and treat it promptly. In this study, children with RMPP had longer hospital stays and duration of fever, more frequent occurrences of pleural effusion, mucus plug, and expulmonary complications, as well as significantly higher levels of CRP and LDH in peripheral blood, compared with NRMPP cases.

Studies have provided the possibility to determine the extent of pneumonia damage by detecting the amount of
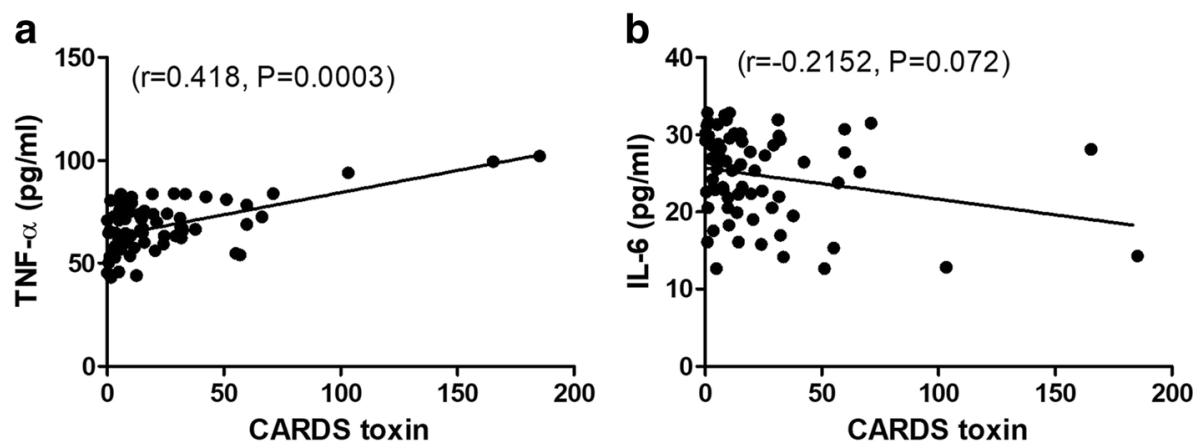

Fig. 2 CARDS toxin was positively correlated with TNF-a level in BALF. The positive correlation between CARDS toxin and TNF-a (a), and a negative correlation between CARDS toxin and IL-6 in BALF from children with MPP (b) 


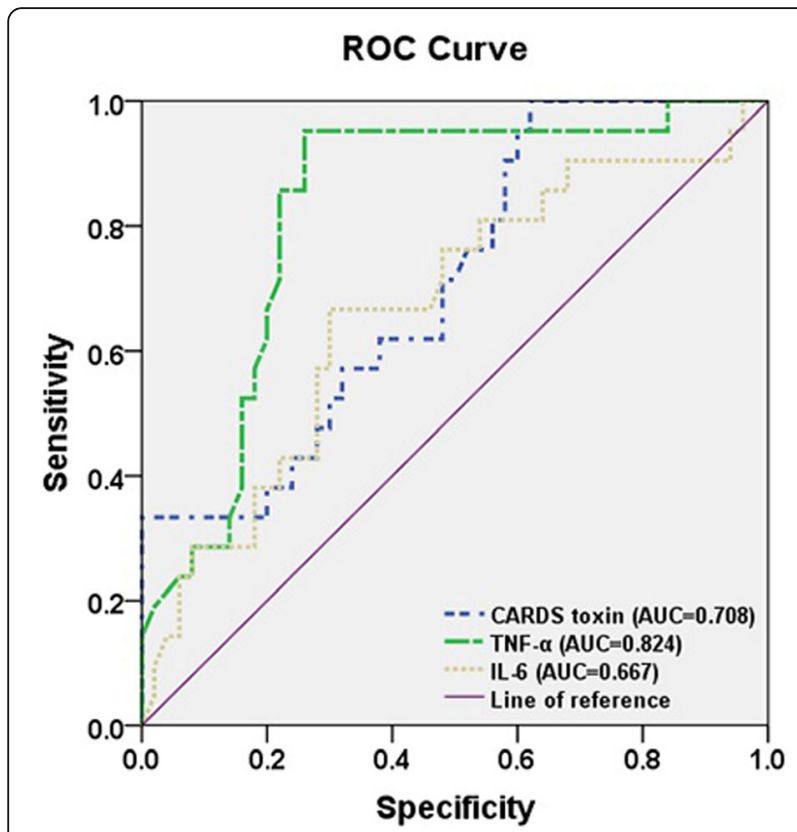

Fig. 3 Diagnostic values of CARDS toxin, TNF-a, and IL-6 in children with RMPP. TNF-a had better diagnostic ability for differentiation of RMPP with the best cut-off of $68.25 \mathrm{pg} / \mathrm{ml}$, AUC of 0.824 and Youden index of 0.692 compared with CARDS toxin and IL-6
CARDS toxin in the clinic.Introduction of recombinant CARDS toxin to the airways of mice caused changes in airway function, cytokine expression, and cellular inflammation, which are consistent with what has been reported for $M$. pneumoniae infection in animal models (Hardy et al., 2009). The concentration of recombinant CARDS toxin in BALF directly correlated with the number of mycoplasma genomes and the degree of histologic pulmonary inflammation and injury in mice (Kannan et al., 2011). However, there are few clinical studies concerning the correlation between CARDS toxin and children with MPP, especially RMPP.

CARDS toxin is a pathogenic virulence factor in $M$. pneumoniae infection (Segovia et al., 2018). In this study, a significant increase in CARDS toxin expression from BALF was observed in both MPP cases compared with control cases and in RMPP cases compared with NRMPP cases. Furthermore, CARDS toxin was significantly higher in BALF from children with MPP with mucus plug than that in children with MPP without plug. Our data reinfore the pathogenic role of CARDS toxin in MPP, especially in RMPP with mucus plug.

Immune disorders caused by $M$. pneumoniae infection are also one of the main factors of RMPP pathogenesis (Chkhaidze \& Kapanadze, 2017; Wang et al., 2014). Introduction of CARDS toxin caused damage to mouse lung tissues, accompanied by the increase of the expression of the pro-inflammatory cytokines (such as TNF- $\alpha$ and IL-6) and immune disorders (Hardy et al., 2009).

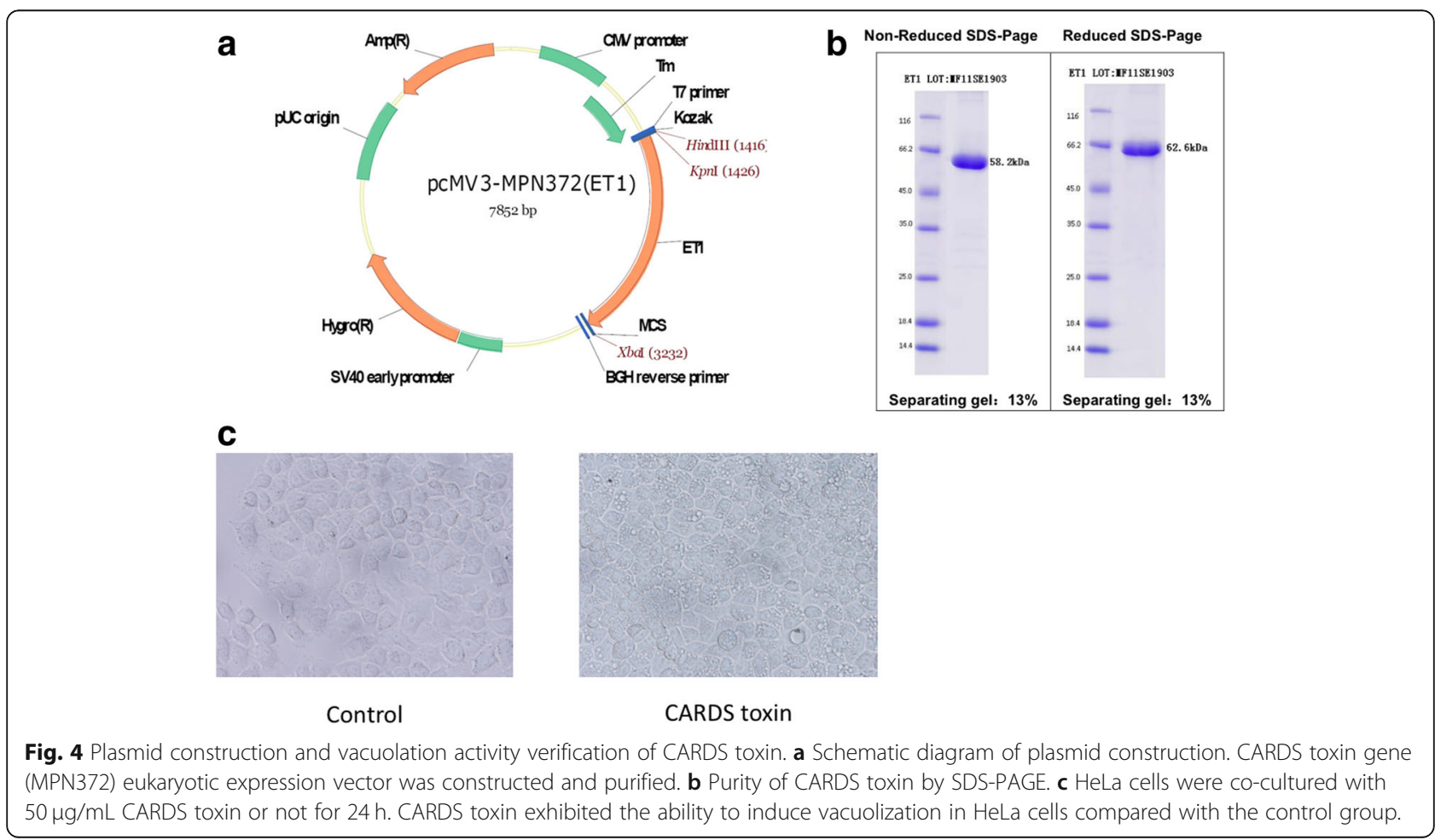



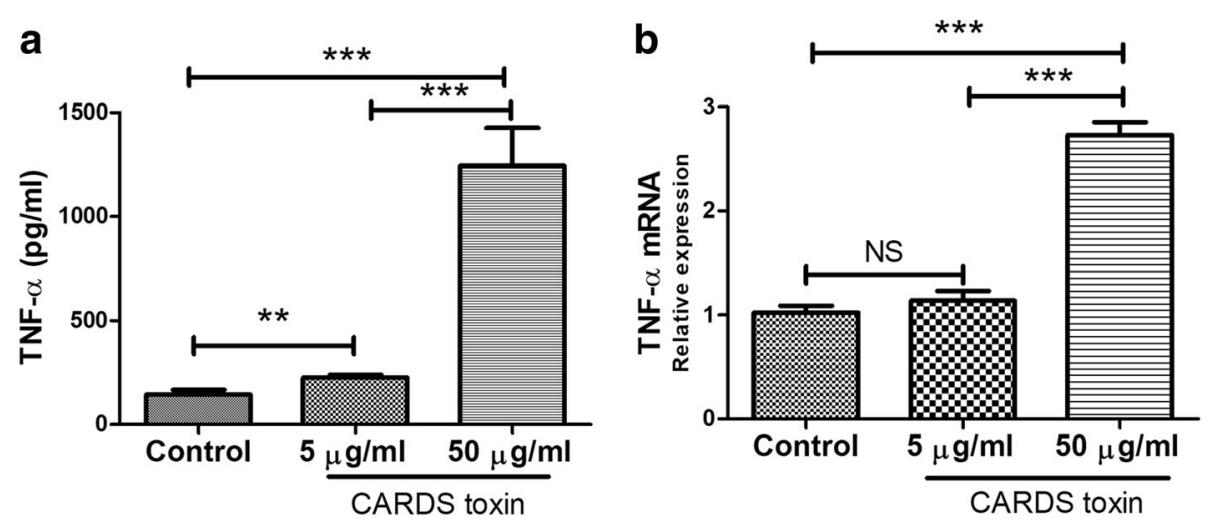

Fig. 5 CARDS toxin induced RAW264.7 macrophages to secrete TNF-a. RAW264.7 macrophages were co-cultured with different concentrations of CARDS toxin $(0,5$, and $50 \mu \mathrm{g} / \mathrm{mL})$ for $48 \mathrm{~h}$. a TNF-a level secreted from RAW264.7 cells was determined by ELISA. b TNF-a mRNA level in RAW264.7 cells was determined by real-time PCR. NS, no significance, ${ }^{* *} p<0.01,{ }^{* * * *} p<0.001$

These data indicated the potential association between CARDS toxin and cytokines and their pathogenic roles in MPP. Therefore, in this study, we explored the correlation between CARDS toxin and cytokines in BALF to further understand the immune status after $M$. pneumoniae infection. Here, we observed significantly higher levels of CARDS toxin, TNF- $\alpha$, and IL- 6 in BALF from both MPP cases compared with control cases and in RMPP cases compared with NRMPP cases.
Furthermore, CARDS toxin was positively correlated with TNF- $\alpha$ level and negatively correlated with IL-6 level in BALF from MPP cases. Importantly, we evaluated the diagnostic values of CARDS toxin, TNF- $\alpha$, and IL-6 in RMPP. Our ROC analysis showed that TNF- $\alpha$ had better diagnostic ability for differentiation of RMPP with the best cut-off of $68.25 \mathrm{pg} / \mathrm{ml}$, AUC of 0.824 and Youden index of 0.692 compared with CARDS toxin and IL-6. Altogether, these data indicated
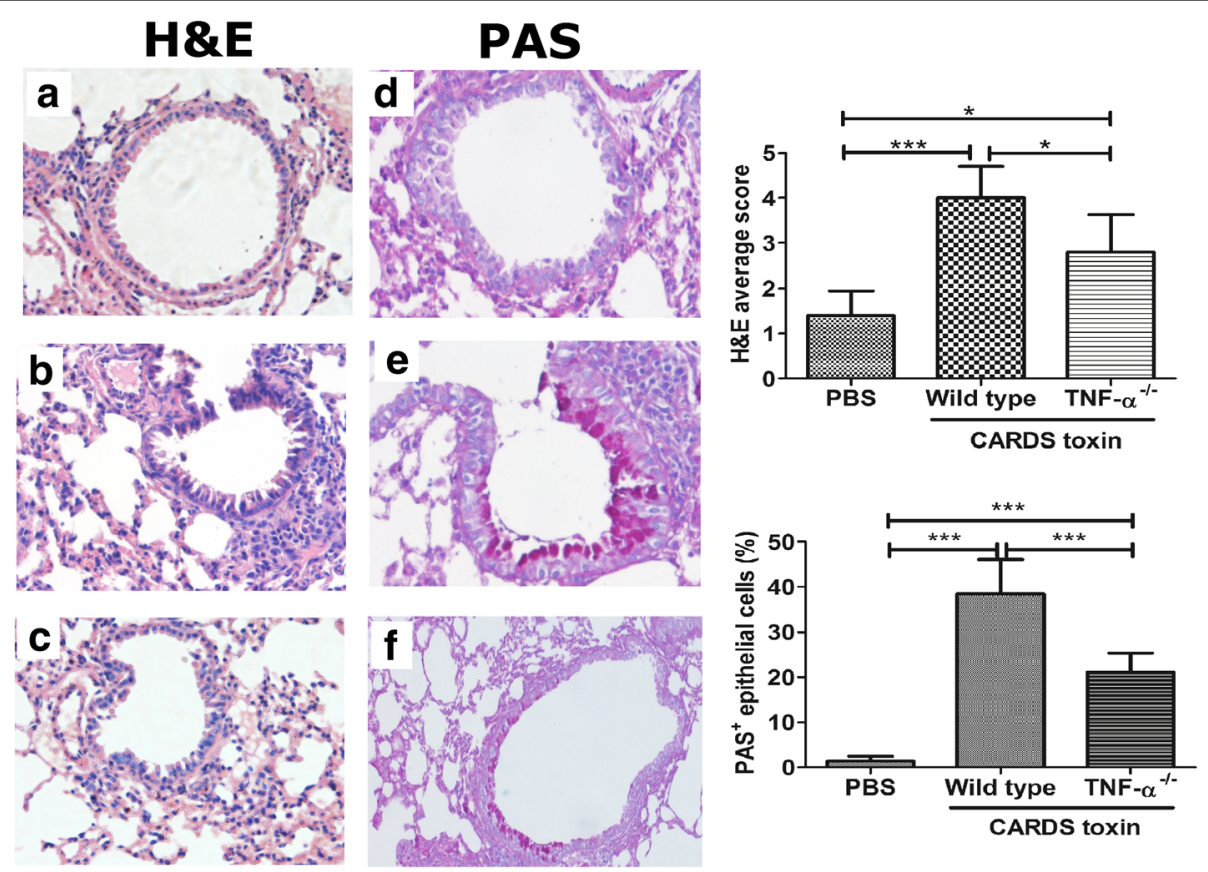

Fig. 6 Effect of CARDS toxin on inflammatory cell infiltration and mucus secretion in mouse lungs. Following intranasal instillation of CARDS toxin (50 $\mu \mathrm{g} /$ $0.5 \mathrm{~mL}$ ) or equivalent PBS, the mouse lung tissues were harvested for heamatoxylin and eosin (H\&E) staining and Periodic acid-Schiff (PAS) staining on the 7th day. Ten bronchial tubes with an inner diameter of 150-200 $\mu \mathrm{m}$ were randomly selected from each section under a light microscope. H\&E average score and the percentage of PAS-positive $\left(\mathrm{PAS}^{+}\right)$epithelial cells in the total number of epithelial cells were analyzed by a pathologist in a blinded fashion. $\mathrm{N}=5$ /group. ${ }^{*} p<0.05,{ }^{* * * *} p<0.001$. $\mathbf{a}$ H\&E staining in PBS controls; $\mathbf{b}$ H\&E staining in CARDS toxin groups; $\mathbf{c}$ H\&E staining in TNF-- ${ }^{-/-}$mice stimulated with CARDS toxin; $\mathbf{d}$ PAS staining in PBS controls; e PAS staining in CARDS toxin groups; $\mathbf{f}$ PAS staining in TNF-a-/- mice stimulated with CARDS toxin 
that high expression of TNF- $\alpha$ may serve as a good predictor for RMPP.

In line with this, a recent study showed that the serum TNF- $\alpha$ level in children with MPP was significantly higher than that in healthy children (Wang et al., 2005). Furthermore, Wang et al. (Ding et al., 2018; Wang et al., 2014) observed that serum TNF- $\alpha$ levels in children with RMPP were significantly higher than those in children with NRMPP, which was confirmed in a mouse model of MPP (Hsia et al., 2012).

Our findings showing the positive correlation between CARDS toxin and TNF- $\alpha$ expression in BALF from all 71 MPP cases suggested that high expression of TNF- $\alpha$ in BALF may be induced by a high amount of CARDS toxin. To address this, we constructed recombinant CARDS toxin and verified its vacuolation activity in HeLa cells. Furthermore, our results revealed that recombinant CARDS toxin induced RAW264.7 macrophages to secrete TNF- $\alpha$ in a dose-dependent manner, whereas it had no significant effect on levels of the other cytokines. Finally, we verified in vivo, the role of CARDS toxin-TNF- $\alpha$ axis in lung inflammatory cell infiltration and mucus secretion. Our results demonstrated that TNF- $\alpha$ deletion partially abrogated the recombinant CARDS toxin-mediated induction of inflammatory cell infiltration and mucus secretion in mouse lungs. Additionally, the level of TNF- $\alpha$ in BALF has been shown to increase with increasing CARDS toxin exposure in mice (Hardy et al., 2009). Collectively, these findings indicated CARDS toxin induced TNF- $\alpha$ expression and thereby involved in enhancing lung inflammatory cell infiltration and mucus secretion.

\section{Conclusions}

The TNF- $\alpha$ level is increased in BALF from children with RMPP and is a good diagnostic biomarker for differentiating RMPP and NRMPP. The high expression of TNF- $\alpha$ may be induced by CARDS toxin. This study provides a new basis for the early diagnosis and treatment of RMPP.

\begin{abstract}
Abbreviations
BALF: Bronchoalveolar lavage fluid; CAP: Community-acquired pneumonia; CARDS: Community-acquired respiratory distress syndrome; CRP: C-reactive protein; ELISA: Enzyme-linked immunosorbent assay; IFN- $\gamma$ : Interferon- $\gamma$; IL: Interleukin; LDH: Lactate dehydrogenase; M. pneumoniae: Mycoplasma pneumoniae; MPP: Mycoplasma pneumoniae pneumonia; NRMPP: Nonrefractory $M$. pneumoniae pneumonia; PCR: Polymerase chain reaction; RMPP: Refractory M. pneumoniae pneumonia; TNF: Tumor necrosis factor
\end{abstract}

\section{Acknowledgements}

We gratefully acknowledge and thank Dr. Hui Tao for her assistance in data collection.

\section{Authors' contributions}

Gang Li, Liping Fan, and Yongdong Yan wrote the main manuscript text; Li Huang, Meijuan Wang, Canhong Zhu, Yuqing Wang, Chuangli Hao, Hansi Liang and Wei Ji collected andanalyzed clinical data; Zhengrong Chen are to take responsibility for study design. All authors reviewed the manuscript. All authors read and approved the final manuscript.

\section{Funding}

The design of the study, collection and analysis of data in this study was supported by the National Natural Science Foundation of China (grant NO. 81870006; 81771676; 31400738); Natural Science Foundation of Jiangsu Province (BK20160349); Science and Technology Program of Suzhou (grant NO. SS201869; SYS201640); Science and Technology Projects of Chinese Traditional Medicine Bureau of Jiangsu Province (grant NO. YB2015176); Social Development Projects of Jiangsu Province (grant NO. BE2016676; BE2019671); Jiangsu Provincial Medical Youth Talent (grant NO.

QNRC2016766; QNRC2016770); Suzhou Medical Youth Talent (grant NO. GSWS2019047); Key Lab of Respiratory Disease of Suzhou (grant NO. SZS201714)

\section{Availability of data and materials}

The datasets generated during and/or analysed during the current study are available from the corresponding author on reasonable request.

\section{Ethics approval and consent to participate}

All procedures performed in studies involving human participants were in accordance with the ethical standards of the Institutional Review Board of Children's Hospital of Soochow University (project approval number 2014LW123) and with the 1964 Helsinki declaration and its later amendments or comparable ethical standards. Participant consent was written by the adults or the legal representatives of patients aged $<16$ years and informed consent forms were archived.

\section{Consent for publication}

Not applicable.

\section{Competing interests}

The authors declare that they have no competing interests.

Received: 22 January 2019 Accepted: 23 July 2019

Published online: 09 August 2019

\section{References}

Azumagawa K, Kambara Y, Murata T, Tamai H. Four cases of arthritis associated with mycoplasma pneumoniae infection. Pediatr Int. 2008;50(4):511-3.

Barreira ER, Souza DC, Goes PF, Bousso A. Septic shock, necrotizing pneumonitis, and meningoencephalitis caused by mycoplasma pneumoniae in a child: a case report. Clin Pediatr. 2009;48(3):320-2.

Becker A, Kannan TR, Taylor AB, Pakhomova ON, Zhang Y, Somarajan SR, Galaleldeen A, Holloway SP, Baseman JB, Hart PJ. Structure of CARDS toxin, a unique ADP-ribosylating and vacuolating cytotoxin from mycoplasma pneumoniae. Proc Natl Acad Sci U S A. 2015;112(16):5165-70.

Chkhaidze I, Kapanadze N. Cytokines as the predictors of severe mycoplasma pneumoniae pneumonia in children. Georgian Med News. 2017;267:89-95.

Ding Y, Chu C, Li Y, Li G, Lei X, Zhou W, Chen Z. High expression of HMGB1 in children with refractory mycoplasma pneumoniae pneumonia. BMC Infect Dis. 2018;18(1):439.

Gao M, Wang K, Yang M, Meng F, Lu R, Zhuang H, Cheng G, Wang X. Transcriptome analysis of Bronchoalveolar lavage fluid from children with mycoplasma pneumoniae pneumonia reveals natural killer and T cellproliferation responses. Front Immunol. 2018;9:1403.

Hardy RD, Coalson JJ, Peters J, Chaparro A, Techasaensiri C, Cantwell AM, Kannan TR, Baseman JB, Dube PH. Analysis of pulmonary inflammation and function in the mouse and baboon after exposure to mycoplasma pneumoniae CARDS toxin. PLoS One. 2009;4(10):e7562.

Hsia BJ, Ledford JG, Potts-Kant EN, Nikam VS, Lugogo NL, Foster WM, Kraft M Abraham SN, Wright JR. Mast cell TNF receptors regulate responses to mycoplasma pneumoniae in surfactant protein a (SP-A) ${ }^{-/-}$mice. J Allergy Clin Immunol. 2012;130(1):205-214.e202.

Kannan TR, Coalson JJ, Cagle M, Musatovova O, Hardy RD, Baseman JB. Synthesis and distribution of CARDS toxin during mycoplasma pneumoniae infection in a murine model. J Infect Dis. 2011;204(10):1596-604.

Liu TY, Lee WJ, Tsai CM, Kuo KC, Lee CH, Hsieh KS, Chang CH, Su YT, Niu CK, Yu HR. Serum lactate dehydrogenase isoenzymes 4 plus 5 is a better biomarker than total lactate dehydrogenase for refractory mycoplasma pneumoniae pneumonia in children. Pediatr Neonatol. 2018;59(5):501-6.

Ma L, Shen CA, Gao L, Li DW, Shang YR, Yin K, Zhao DX, Cheng WF, Quan DQ. Anti-inflammatory activity of chitosan nanoparticles carrying NF-kappaB/p65 
antisense oligonucleotide in RAW264.7 macropghage stimulated by lipopolysaccharide. Colloids Surf B Biointerfaces. 2016;142:297-306.

Principi N, Esposito S. Biomarkers in pediatric community-acquired pneumonia. Int J Mol Sci. 2017; 18(2). pii: E447.

Ramasamy K, Balasubramanian S, Manickam K, Pandranki L, Taylor AB, Hart PJ, Baseman JB, Kannan TR. (2018) Mycoplasma pneumoniae community-acquired respiratory distress syndrome toxin uses a novel KELED sequence for retrograde transport and subsequent cytotoxicity. mBio. 9(1). pii: e01663-e01617.

Segovia JA, Chang TH, Winter VT, Coalson JJ, Cagle MP, Pandranki L, Bose S, Baseman JB, Kannan TR. (2018) NLRP3 Is a Critical Regulator of Inflammation and Innate Immune Cell Response during Mycoplasma pneumoniae Infection. Infect Immun. 86(1). pii: e00548-e00517.

Shimizu T. Inflammation-inducing factors of mycoplasma pneumoniae. Front Immunol. 2016;7:414.

Sungurlu S, Balk RA. The role of biomarkers in the diagnosis and Management of Pneumonia. Clin Chest Med. 2018;39(4):691-701.

Tamura A, Matsubara K, Tanaka T, Nigami H, Yura K, Fukaya T. Methylprednisolone pulse therapy for refractory mycoplasma pneumoniae pneumonia in children. J Inf Secur. 2008;57(3):223-8.

Wang K, Gao M, Yang M, Meng F, Li D, Lu R, Wang Y, Zhuang H, Li M, Cheng G, Wang $X$. Transcriptome analysis of bronchoalveolar lavage fluid from children with severe mycoplasma pneumoniae pneumonia reveals novel gene expression and immunodeficiency. Hum Genomics. 2017a;11(1):4-4.

Wang L, Lu S, Feng Z, Li L, Niu B, Shuai J, Cao L, Li G, Liu J. The early examination of combined serum and imaging data under flexible fiberoptic bronchoscopy as a novel predictor for refractory mycoplasma pneumoniae pneumonia diagnosis. Medicine. 2017b;96(50):e9364.

Wang M, Wang Y, Yan Y, Zhu C, Huang L, Shao X, Xu J, Zhu H, Sun X, Ji W. Clinical and laboratory profiles of refractory mycoplasma pneumoniae pneumonia in children. Int J Infect Dis. 2014;29:18-23.

Wang Y, Zhang Y, Lu W, Wang L. Serum tumor necrosis factor-alpha and interferon-gamma levels in pediatric mycoplasma pneumoniae pneumonia: a systematic review and meta-analysis. Can Respir J. 2005;2018:8354892.

Xu D, Li S, Chen Z, Du L. Detection of mycoplasma pneumoniae in different respiratory specimens. Eur J Pediatr. 2011;170(7):851-8.

Zhang Y, Zhou Y, Li S, Yang D, Wu X, Chen Z. The clinical characteristics and predictors of refractory mycoplasma pneumoniae pneumonia in children. PLoS One. 2016;11(5):e0156465.

\section{Publisher's Note}

Springer Nature remains neutral with regard to jurisdictional claims in published maps and institutional affiliations.

Ready to submit your research? Choose BMC and benefit from:

- fast, convenient online submission

- thorough peer review by experienced researchers in your field

- rapid publication on acceptance

- support for research data, including large and complex data types

- gold Open Access which fosters wider collaboration and increased citations

- maximum visibility for your research: over $100 \mathrm{M}$ website views per year

At $\mathrm{BMC}$, research is always in progress.

Learn more biomedcentral.com/submissions 